Сравнительная оценка различных схем периоперационной антикоагулянтной терапии у пациентов с фибрилляцией предсердий, нуждающихся в плановом хирургическом лечении

\author{
Уддин $\Lambda$. Н., Габитова М.А., Соколова А.А., Морозова Н.С., Напалков А. А., \\ Вычужанин А.В., Егоров А. В., Фомин В. В. \\ ФГАОУ ВО Первый Московский государственный медицинский университет имени И. М. Сеченова \\ Минздрава России (Сеченовский Университет). Москва, Россия
}

\begin{abstract}
Изучены литературные данные о применении различных схем периоперационной антикоагулянтной терапии (АКТ) у больных с фибрилляцией предсердий при проведении планового хирургического лечения. Отмечено, что врачи нередко сталкиваются с ситуацией, когда пациентам, получающим АКТ, показаны инвазивные исследования и хирургические вмешательства. Периоперационное ведение такой категории больных является достаточно сложным, поскольку, с одной стороны, хирургические вмешательства на фоне АКТ ассоциируются с высоким риском развития интраоперационных геморрагических осложнений, а, с другой стороны, прекращение АКТ может увеличить риск развития тромбоэмболических осложнений. Рассмотрены варианты перипроцедурной АКТ у пациентов, получающих антагонисты витамина К. Отмечено, что в качестве альтернативы постоянной AKT в настоящее время используется переходная терапия ("мосттерапия"), заключающаяся в отмене перорального антикоагулянта
\end{abstract}

с назначением низкомолекулярных или нефракционированных гепаринов, целью которой является снижение риска кровотечения при адекватной тромбопрофилактике. Приведены результаты клинических исследований, посвященных оценке применения "мосттерапии" при оперативных вмешательствах. Представлены варианты стратификации риска тромбоэмболических и геморрагических осложнений при хирургических операциях.

Ключевые слова: фибрилляция предсердий, антикоагулянтная терапия, кровотечения, тромбоэмболические осложнения, мосттерапия.

Кардиоваскулярная терапия и профилактика. 2018;17(3):71-78 http://dx.doi.org/10.15829/1728-8800-2018-3-71-78

Поступила 28/12-2017

Принята к публикации 05/03-2018

\title{
Comparison of schemes of perioperational anticoagulation in atrial fibrillation patients demanding for surgery
}

Uddin L. N., Gabitova M.A., Sokolova A.A., Morozova N. S., Napalkov D. A., Vychuzhanin D. V., Egorov A. V., Fomin V.V.

I. M. Sechenov First Moscow State Medical University of the Ministry of Health. Moscow, Russia

A literature review provided, on the usage of various schemes of perioperational anticoagulation therapy (ACT) in atrial fibrillation patients undergoing scheduled surgery. It is noted that clinicians quite often pass through a situation when patients taking ACT require invasive investigations and surgery. Perioperational management of such category of patients is complicated as, on the one hand, the surgery under ACT is associated with intraoperational hemorrhagic complications, and, on the other hand, ACT cessation might increase the risk of thrombotic complications. The variants assessed, of different periprocedural ACT in patients taking vitamin $\mathrm{K}$ antagonists. It was found that as an alternative to continuous ACT recently, bridge therapy with low molecular weight heparins applied, aiming the decrease of the risk of bleeding in adequate thromboprophylaxis. The results of clinical trials provided on the assessment of bridge therapy in surgery. Risk stratification approaches presents for thromboembolic and hemorrhagic complications in surgery.

Keywords: atrial fibrillation, anticoagulation, bleedings, thromboembolic complications, bridge therapy.

Cardiovascular Therapy and Prevention. 2018;17(3):71-78

http://dx.doi.org/10.15829/1728-8800-2018-3-71-78

АВК - антагонисты витамина К, АКТ - антикоагулянтная терапия, МНО - международное нормализованное отношение, НМГ- низкомолекулярный гепарин, НФГ - нефракционированный гепарин, ТЭЛА тромбоэмболия легочной артерии, ТЭО - тромбоэмболические осложнения, ФП - фибрилляция предсердий, ТГВ - тромбоз глубоких вен, ОАК - оральные антикоагулянты, ППОАК - прямые пероральные антикоагулянты, BRIDGE - Bridging Anticoagulation in Patients who Require Temporary Interruption of Warfarin Therapy for an Elective Invasive Procedure or Surgery, $\mathrm{CHA}_{2} \mathrm{DS}_{2}$-VASc - Congestive Heart failure, Hypertension ( 1 ball), Age $\geqslant 75$ years ( 2 ball), Diabetes mellitus ( 1 ball), Stroke ( 2 ball), Vascular disease, Age 65-74 years, Sex category ( 1 ball) - шкала для оценки риска тромбоэмболических осложнений у больных с фибрилляцией предсердий, CHADS 2 - Congestive heart failure, Hypertension, Age $\geqslant 75$ years, Diabetes mellitus ( 1 ball), Prior Stroke or TIA or Thromboembolism (2 ball); HAS-BLED - Hypertension, Abnormal renal-liver function, Stroke, Bleeding history or predisposition, Labile international normalized ratio, Elderly ( 65 years), Drugs or alcohol concomitantly.

*Автор, ответственный за переписку (Corresponding author):

Тел.: +7 (925) 025-98-10

e-mail: luisiana9090@mail.ru

[Уддин Л. Н. ${ }^{*}$ - аспирант кафедры факультетской терапии № 1, Габитова М. А. - аспирант кафедры факультетской терапии № 1, Соколова А. А. - к. м.н., ассистент кафедры факультетской терапии № 1, заместитель директора Дирекции образовательных программ Международной школы "Медицина будущего", Морозова Н.С. - к.м.н., доцент кафедры факультетской терапии № 1, Напалков Д. А. - Д. м.Н., профессор кафедры факультетской терапии № 1, Вычужанин Д. В. - к. М.н., врач-онколог онкологического хирургического отделения, Егоров А. В. - Д.м.н., профессор, зав. отделением онкологического хирургического отделения, Фомин В. В. - д.м.н., профессор, член-корр. РАН, проректор по лечебной работе, заведующий кафедрой факультетской терапии № 1]. 


\section{Введение}

Фибрилляция предсердий (ФП) в настоящее время является актуальной проблемой современной кардиологии, а также значимым фактором роста расходов на здравоохранение [1, 2]. Для этого, часто развивающегося, нарушения ритма сердца характерен высокий риск развития инсульта и системных эмболий, в связи с чем ФП существенно влияет на продолжительность и качество жизни пациентов [2-4]. В РФ проживают не менее 2,5 млн пациентов с ФП [2, 3]. Максимальные уровни заболеваемости и распространенности ФП отмечаются в индустриально развитых странах. В 2010г в мире насчитывалось 20,9 млн мужчин и 12,6 млн женщин, страдающих ФП, а к 2030г в странах Европейского Союза прогнозируется увеличение количества данной категории больных на 14-17 млн. Высокая распространенность ФП может быть объяснена как увеличением частоты диагностики ее бессимптомной формы, так и увеличением средней продолжительности жизни и, соответственно, распространенности состояний, предрасполагающих к развитию ФП $[4,5]$. Эта аритмия часто сочетается с артериальной гипертензией (73\%), ишемической болезнью сердца $(65,2 \%)$, в т.ч. инфарктом миокарда $(6,9 \%)$, и сахарным диабетом (до 9\%) [2, 3].

В настоящее время общепризнанно, что для профилактики тромбоэмболических осложнений (ТЭО) больным с ФП показано назначение антикоагулянтной терапии (AKT). При этом врачи нередко сталкиваются с ситуацией, когда пациентам, получающим AKT, показаны необходимые инвазивные исследования и хирургические вмешательства $[1,6]$. Периоперационное ведение такой категории больных является достаточно сложным, поскольку, с одной стороны, хирургические вмешательства на фоне АКТ ассоциируются с высоким риском развития интраоперационных геморрагических осложнений, a, с другой стороны, прекращение АКТ может увеличить риск развития ТЭО.

Таким образом, весьма актуален анализ современных представлений о возможности проведения периоперационной АКТ у пациентов с ФП при плановых хирургических вмешательствах.

В настоящее время, в клинической практике с целью профилактики и лечения тромбоза или ТЭО наиболее распространено назначение следующих пероральных антикоагулянтов: антагонисты витамина К (АВК) (варфарин), прямые пероральные антикоагулянты (ривароксабан, дабигатран, апиксабан).

Варианты перипроцедурной АKT у пациентов, получающих АВК

В настоящее время в клинической практике используются следующие подходы к ведению пациентов, получающих АКТ в периоперационный период:
- отмена АКТ перед выполнением операции с последующим возобновлением терапии в послеоперационном периоде;

- “мост-терапия" - переходная терапия, заключающаяся в отмене перорального антикоагулянта с переходом на низкомолекулярные гепарины (НМГ) или нефракционированные гепарины (НФГ) перед процедурой и затем обратным переводом на исходный препарат;

- проведение оперативного лечения без отмены AKT.

Согласно рекомендациям Европейского общества кардиологов (ESC) по предоперационному обследованию и ведению пациентов при выполнении внесердечных хирургических вмешательств (2014) у пациентов, принимающих АВК, следует относить к категории высокого риска развития пери- и послеоперационных геморрагических осложнений. Таким больным рекомендуется прекращение приема ABK за 3-5 сут. перед выполнением хирургического вмешательства с последующим ежедневным контролем уровня международного нормализованного отношения (МНО) до достижения целевых значений этого показателя $\leqslant 1,5$. При выполнении этого условия хирургическая операция может рассматриваться как безопасная с точки зрения риска кровотечений $[7,8]$.

Следует отметить, что временная отмена постоянной пероральной АКТ у пациентов с высоким риском ТЭО может повлечь за собой развитие инсульта или системной эмболии. В связи с этим в качестве альтернативы постоянной АКТ АВК рассматривают переходную терапию (“мост-терапию”), включающую назначение НФГ или НМГ. Схема “мост-терапии" представлена на рисунке 1.

"Мост-терапия" может быть начата через сут. после отмены АВК или позднее при МНО <2. При этом внутривенную инфузию НФГ следует прекратить за 4-6 ч до операции, а последняя инъекция НМГ должна выполняться не менее чем за 24 ч до начала хирургического вмешательства [9]. Целью "мост-терапии" является снижение риска кровотечения с сохранением должной тромбопрофилактики.

Безусловно, перед выполнением плановой операции у больного, принимающего варфарин, необходимо соотнести риски развития геморрагических осложнений и ТЭО. Последние потенциально преобладают у пациентов с ФП при значении показателя шкалы $\mathrm{CHA}_{2} \mathrm{DS}_{2}$-VASc (Congestive Heart failure, Hypertension, Age (2 ball), Diabetes mellitus, Stroke (2 ball), Vascular disease, Age, Sex category - шкала для оценки риска ТЭО у больных с ФП) $\geqslant 4$, при наличии механических клапанов сердца либо недавно (до 3-6 мес.) имплантированных биологических клапанов, после пластики митрального клапана в течение 3 мес., при недавно перенесенной (за 3 
последних мес.) тромбоэмболии легочной артерии (ТЭЛА), а также при тромбофилиях [9, 10]. Параметры шкалы $\mathrm{CHA}_{2} \mathrm{DS}_{2}$-VASc представлены в таблице 1.

Следует учитывать вариант планируемого хирургического вмешательства, которое увеличивает риск развития кровотечения. К операциям с высоким риском геморрагических осложнений относят процедуры, при которых невозможно выполнение компрессии сосудов: в этих случаях временная отмена непрямых антикоагулянтов и переход на использование НМГ являются обязательными.

Необходимым условием предоперационного ведения пациентов, получающих АВК, является контроль уровня МНО в день выполнения операции. При уровне МНО >1,5, рекомендуется отложить либо отменить хирургическое вмешательство.

Возобновление терапии НМГ или НФГ в прежней дозе в послеоперационный период рекомендовано в течение первых 12-24 ч после выполненного вмешательства, однако в отдельных ситуациях возникает необходимость отсрочить возобновление АКТ на 48-72 ч. Это, в первую очередь, относится к вмешательствам, для которых характерны большой объем и высокий риск развития кровотечений:

- катетерная аблация при простой левосторонней суправентрикулярной тахикардии (например, при WPW-синдроме);

- спинальная или эпидуральная анестезия;

- поясничная диагностическая пункция;

- торакальные операции;

- операции на органах брюшной полости;

- крупные ортопедические вмешательства;

- биопсия печени и почек;

- трансуретральная резекция простаты;

- экстракорпоральная ударно-волновая литотрипсия (ESWL) [7, 11].

Как правило, прием пациентом АВК возобновляется на 1-2 сут. после операции при достижении адекватного гемостаза в предоперационной поддерживающей дозе с ее дальнейшим 50\%-ным повыше-

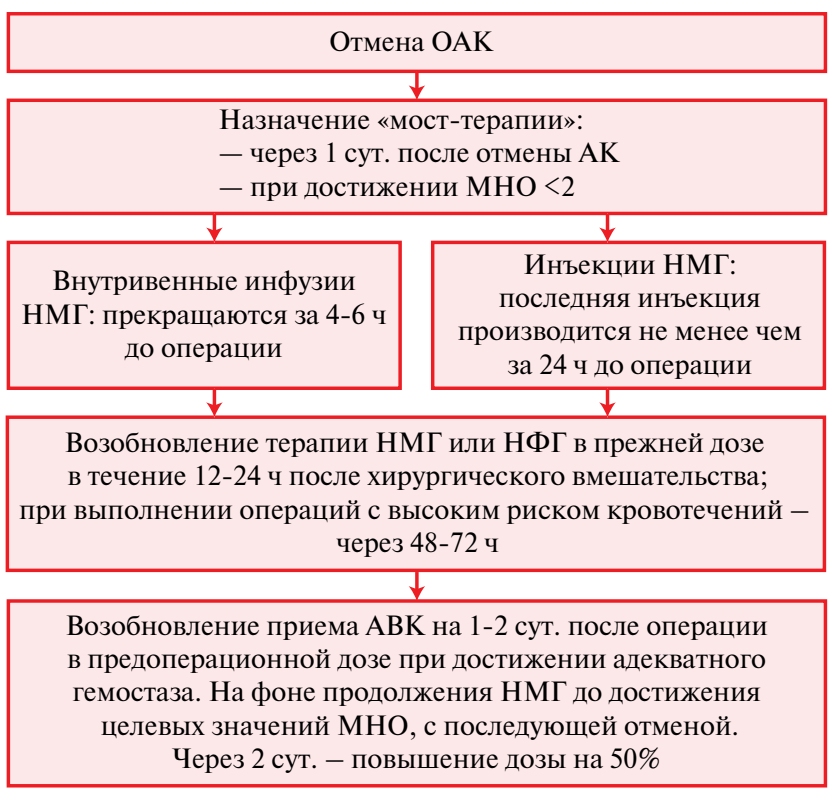

Puc. 1 Схема проведения “мост-терапии”.

нием в течение последующих 2 сут., после чего терапия продолжается. При этом используется стандартная поддерживающая доза НМГ или НФГ, которые отменяются при достижении целевого уровня МНО.

Хирургические и диагностические процедуры, сопровождающиеся риском развития кровотечения, целесообразно выполнять на фоне использования субтерапевтических доз антикоагулянтов продолжительностью до 48 ч без перехода на гепарин, если при этом риск развития ТЭО сохраняется на низком уровне.

Большинство специалистов практикуют перипроцедурное прерывание приема варфарина, хотя результаты последних лет свидетельствуют о том, что в 40-60\% случаев этого делать не требуется. Установлено, что 44\% хирургов при выполнении вмешательств прерывают пероральную АКТ на время выполнения процедур, для которых харак-

Таблища 1

$\mathrm{CHA}_{2} \mathrm{DS}_{2}$-VASc - шкала оценки риска ТЭО у больных с ФП/трепетанием предсердий

\begin{tabular}{ll}
\hline Фактор риска & Баллы \\
\hline Инсульт, транзиторная ишемическая атака или артериальная тромбоэмболия в анамнезе & 2 \\
\hline Возраст $\geqslant 75$ лет & 2 \\
\hline Артериальная гипертензия & 1 \\
\hline Сахарный диабет & 1 \\
\hline Застойная сердечная недостаточность/дисфункция ЛЖ (в частности, ФВ $\leqslant 40 \%)$ & 1 \\
\hline Сосудистое заболевание (инфаркт миокарда в анамнезе, периферический атеросклероз, атеросклеротические бляшки в аорте) & 1 \\
\hline Возраст 65-74 года & 1 \\
\hline Женский пол & 1 \\
\hline
\end{tabular}

Примечание: ЛЖ - левый желудочек, ФВ - фракция выброса. 
Шкала балльной оценки риска развития ТЭО (Caprini)

\begin{tabular}{|c|c|c|c|}
\hline 1 балл & 2 балла & 3 балла & 5 баллов \\
\hline 41-60 лет & 61-74 года & $>74$ лет & Инсульт (<1 мес. назад) \\
\hline Малая операция & Артроскопическая операция & Анамнез ВТЭО & Замена крупного сустава \\
\hline ИМТ $>25 \mathrm{Kг} / \mathrm{M}^{2}$ & $\begin{array}{l}\text { Большая открытая операция } \\
(>50 \text { мин) }\end{array}$ & Семейный анамнез ВТЭО & $\begin{array}{l}\text { Перелом бедра, костей таза, } \\
\text { голени }\end{array}$ \\
\hline Отек нижних конечностей & $\begin{array}{l}\text { Лапароскопическая операция } \\
\text { (>45 мин) }\end{array}$ & Лейденская мутация & $\begin{array}{l}\text { Травма спинного мозга } \\
(<1 \text { мес. назад) }\end{array}$ \\
\hline Варикозное расширение вен & Онкология & Мутация в гене протромбина & - \\
\hline $\begin{array}{l}\text { Беременность или послеродовый } \\
\text { период }\end{array}$ & Постельный режим (>3 сут.) & Волчаночный антикоагулянт & - \\
\hline $\begin{array}{l}\text { Невынашивание беременности } \\
\text { в анамнезе (2-3 триместр) }\end{array}$ & Гипсовая повязка & Антитела к кардиолипину & - \\
\hline Прием эстрогенов/гестагенов & Катетер в центральной вене & $\begin{array}{l}\text { Повышение уровня } \\
\text { гомоцистеина в плазме }\end{array}$ & - \\
\hline Сепсис (<1 мес.) & - & $\begin{array}{l}\text { Гепарининдуцированная } \\
\text { тромбоцитопения }\end{array}$ & - \\
\hline $\begin{array}{l}\text { Тяжелое заболевание легких, } \\
\text { в т.ч. пневмония (<1 мес.) }\end{array}$ & - & Другие тромбофилии & - \\
\hline Нарушение функции дыхания & - & - & - \\
\hline Острый инфаркт миокарда & - & - & - \\
\hline $\begin{array}{l}\text { Застойная сердечная } \\
\text { недостаточность }(<1 \text { мес.) }\end{array}$ & - & - & - \\
\hline $\begin{array}{l}\text { Анамнез воспалительного заболе- } \\
\text { вания кишечника }\end{array}$ & - & - & - \\
\hline $\begin{array}{l}\text { Терапевтический пациент } \\
\text { на постельном режиме }\end{array}$ & - & - & - \\
\hline
\end{tabular}

Примечание: ИМТ - индекс массы тела, ВТЭО - венозные тромбоэмболические осложнения.

терен низкий риск кровотечения. В другом исследовании было показано, что 90-100\% специалистов считают возможным проведение "мост-терапии" при планировании процедур с низким риском кровотечений независимо от риска ТЭО [12].

Таким образом, небольшие по объему инвазивные вмешательства с низким периоперационным риском развития тяжелых кровотечений (экстракция зуба, процедуры на каналах корня зуба, небольшие дерматологические операции, удаление катаракты) можно выполнять без изменений режима антикоагулянтной терапии, однако при этом следует поддерживать МНО на нижней границе терапевтического диапазона, а также использовать местные гемостатические средства.

Стратификация риска ТЭО и геморрагических осложнений при хирургических вмешательствах

Адекватная оценка риска развития послеоперационных венозных ТЭО, включающих тромбоз глубоких вен (ТГВ) и ТЭЛА, является важнейшим условием их эффективной профилактики. При этом стратификация риска может осуществляться с помощью учета наличия у больного состояний, предрасполагающих к развитию тромбоза [13].

На сегодняшний день предложено несколько моделей оценки риска, из которых наиболее удобной и проверенной является шкала Caprini J. (1995) (таблица 2). Эта балльная система учитывает индивидуальные характеристики пациентов и факторы, провоцирующие развитие этих осложнений (особенности основного заболевания и оперативного вмешательства) [14].

Достоверность шкалы Caprini была подтверждена ретроспективными исследованиями с использованием баз данных регистров, включавших 16 тыс. пациентов общехирургического, сосудистого, урологического, оториноларингологического профилей, а также пациентов, находившихся в отделениях интенсивной терапии и перенесших пластические и реконструктивные операции. В ряде исследований была выявлена четкая взаимосвязь между количеством баллов по шкале Caprini и частотой развития венозных ТЭО. Между тем в группах больных с традиционно высоким риском осложнений проспективная оценка клинической ценности этой шкалы до настоящего времени не проводилась.

Установлено, что число баллов по шкале Caprini коррелирует с частотой развития послеоперационных венозных ТЭО у пациентов из групп высокого риска по стандартной стратификации. Показано, что при наличии 11 баллов по данной шкале повышается вероятность развития венозных ТЭО даже у больных, которым проводится их медикаментоз- 
Таблица 3

$\mathrm{CHADS}_{2}$ - шкала оценки риска инсульта у больных с ФП/трепетанием предсердий

\begin{tabular}{ll}
\hline Фактор риска & Баллы \\
\hline Инсульт или транзиторная ишемическая атака в анамнезе & 2 \\
\hline Артериальная гипертензия & 1 \\
\hline Возраст $\geqslant 75$ лет & 1 \\
\hline Сахарный диабет & 1 \\
\hline Умеренное или тяжелое снижение сократимости левого желудочка/недавние симптомы сердечной недостаточности & 1 \\
\hline
\end{tabular}

Таблища 4

HAS-BLED - шкала оценки риска кровотечений

\begin{tabular}{ll}
\hline Факторы риска & Баллы \\
\hline Артериальная гипертензия (систолическое АД >160 мм рт.ст.) & 1 \\
\hline $\begin{array}{l}\text { Нарушенная функция печени (тяжелое хроническое заболевание или повышение билирубина >2 раз от верхней границы } \\
\text { нормы в сочетании с повышенными АСТ/АЛТ >3 раз от верхней границы нормы) }\end{array}$ & 1 \\
\hline Нарушенная функция почек (диализ, трансплантация или креатинин श200 мкмоль/л) & 1 \\
\hline Инсульт & 1 \\
\hline Кровотечение в анамнезе и/или предрасположенность к кровотечениям (в т.ч. анемия) & 1 \\
\hline Лабильное МНО (нестабильное/высокое или в терапевтическом диапазоне & 1 \\
\hline Возраст >65 лет & 1 \\
\hline Злоупотребление алкоголем & 1 \\
\hline Прием лекарств, повышающих риск кровотечения (антиагреганты, НПВС) & 1 \\
\hline
\end{tabular}

Примечание: АД - артериальное давление, АСТ/АЛТ - аспартатаминотрансфераза/аланинаминотрансфераза, НПВС - нестероидные противовоспалительные средства.

ная профилактика, что позволило выделить эту категорию пациентов в группу крайне высокого риска развития ТЭО [14].

Риск развития ТЭО у пациентов с ФП оценивается с помощью шкал $\mathrm{CHADS}_{2}$ (таблица 3) и $\mathrm{CHA}_{2} \mathrm{DS}_{2}$-VASc. В частности, шкала $\mathrm{CHA}_{2} \mathrm{DS}_{2}-$ $\mathrm{VASc}$ позволяет осуществлять более точную оценку, чем $\mathrm{CHADS}_{2}$, например, для выявления больных с низким риском тромбоэмболических событий $\left(\mathrm{CHADS}_{2}=0\right)$. Тем не менее, оценка по шкале $\mathrm{CHADS}_{2}$ остается более надежной при решении о целесообразности проведения "мосттерапии".

С учетом рекомендаций Американского Общества Торакальных Хирургов 2012г пациентам с 0-2 баллами по шкале $\mathrm{CHADS}_{2}$ не требуется "мосттерапия", тогда как у больных с 5-6 баллами "мосттерапия" возможно целесообразна. Для пациентов с 3-4 баллами по шкале CHADS $_{2}$ решение о необходимости "мост-терапии" остается на усмотрение лечащего врача. В публикациях последних лет авторы предлагают использовать “мост-терапию" у пациентов с ФП и оценкой $\geqslant 2$ баллов по шкале $\mathrm{CHA}_{2} \mathrm{DS}_{2}$-VASc.

В настоящее время для оценки риска кровотечений на фоне пероральной АКТ в клинической практике наиболее часто используется шкала HASBLED (таблица 4). Она также может быть использована для оценки риска кровотечений при решении вопроса о проведении “мост-терапии”.
В периоперационном периоде соотношение рисков развития кровотечений и ТЭО составляет $\sim 13: 1$ при применении "мост-терапии" и 5:1 без ее проведения [6, 15, 16].

Современные данные о применении "мост-терапии" при оперативных вмешательствах

В ретроспективном исследовании были проанализированы результаты лечения 1812 пациентов, принимавших $\mathrm{ABK}$ и прекративших прием на время проведения оперативного вмешательства. Оказалось, что $73 \%$ пациентов, которым проводилась “мост-терапия” для предотвращения ТЭО, имели низкий риск их развития [17]. Этот анализ показал, что для современной клинической практики характерен достаточно низкий уровень настороженности специалистов в отношении назначения "мост-терапии" при лечении антикоагулянтами: пациентам с умеренным и даже низким риском ТЭО переходная терапия назначается “на всякий случай” [17].

При анализе результатов, полученных у 2803 пациентов с ФП, включенных в регистр ORBIT-AF (Outcomes Registry for Better Informed Treatment for Atrial Fibrillation), у которых АКТ в периоперационный период прерывалась, было отмечено, что переходную терапию пациентам назначали без учета каких-либо строгих критериев [18].

В рандомизированном, двойном слепом, плацебо-контролируемом исследовании BRIDGE (Bridging Anticoagulation in Patients who Require Temporary Interruption of Warfarin Therapy for an 
Сроки временной отмены препаратов в зависимости от уровня риска кровотечения и клиренса креатинина

\begin{tabular}{lllll}
\hline \multirow{2}{*}{$\begin{array}{l}\text { Клиренс креатинина } \\
\text { мл/мин }\end{array}$} & \multicolumn{2}{c}{ Дабигатран } & \multicolumn{2}{c}{ Апиксабан-эндоксабан-ривароксабан } \\
\cline { 2 - 5 } & Низкий риск & Высокий риск & Низкий риск & Высокий риск \\
\hline$\geqslant 80$ & $\geqslant 24$ ч & $\geqslant 48$ ч & $\geqslant 24$ ч & $\geqslant 48$ ч \\
\hline $50-80$ & $\geqslant 36$ ч & $\geqslant 72$ ч & $\geqslant 24$ ч & $\geqslant 48$ ч \\
\hline $30-50$ & $\geqslant 48$ ч & $\geqslant 96$ ч & $\geqslant 24$ ч & $\geqslant 48$ ч \\
\hline $15-30$ & Не показан & Не показан & \\
\hline$<15$ & Нет официальных показаний к применению & & \\
\hline
\end{tabular}

Elective Invasive Procedure or Surgery) 1884 пациента с ФП были распределены в группы перипроцедурной переходной АКТ НМГ, далтепарином или плацебо. Причины прерывания приема оральных антикоагулянтов (ОАК) не были указаны, однако важно отметить, что у 89,4\% пациентов выполнялись вмешательства с потенциально низким риском кровотечения. Среднее число баллов по шкале $\mathrm{CHADS}_{2}$ составило 2,3, т.е. в исследуемой популяции наблюдался преимущественно умеренный риск ТЭО. Основными конечными точками в этом исследовании были частота ТЭО и больших кровотечений. Было показано, что частота инсультов и системных эмболий в группе плацебо не превышала таковую в группе “мост-терапии” - 0,4\% vs $0,3 \%(\mathrm{p}=0,01)$ для не меньшей эффективности, но при этом частота больших и малых кровотечений у пациентов в группе плацебо была достоверно ниже, чем во всей выборке больных, получавших "мост-терапию” - $1,3 \%$ vs $3,2 \%(p=0,005)$ и $12,0 \%$ vs $20,9 \%$ $(\mathrm{p}=0,001)$, соответственно. Межгрупповые различия по частоте инфаркта миокарда, ТГВ, ТЭЛА и летальных исходов отсутствовали [19].

Результаты исследования показали, что у пациентов без “мост-терапии" не наблюдалось роста числа ТЭО, но при этом число кровотечений у них было меньше. Важным ограничением исследования BRIDGE было то, что в выборку были включены пациенты с умеренным риском развития ТЭО, для которых показанием к проведению АКТ являлась ФП. В связи с этим ряд авторов считают, что следует с осторожностью экстраполировать результаты исследования BRIDGE на группы больных с более высоким риском ТЭО, в частности, пациентов с ФП и более высокими уровнями шкалы $\mathrm{CHADS}_{2}$, с протезами клапанов сердца, а также у лиц с венозными или артериальными тромбозами в анамнезе.

$\mathrm{K}$ настоящему времени на основании накопленных данных сформулирован современный подход к назначению периоперационной АКТ. Полагают, что, прежде всего, по возможности следует избегать прерывания приема ОАК. Кандидатами для непрерывной АКТ являются пациенты с умеренным или высоким риском развития ТЭО, кото- рым выполняются операции с относительно низким риском кровотечения. Для снижения риска развития кровотечения у пациентов при проведении непрерывной АКТ следует рассмотреть возможность снижения целевого МНО до 2,0 в периоперационный период. При необходимости прерывания AКТ у больных с низким или умеренным риском ТЭО рекомендуется избегать назначения "мосттерапии” [20].

Как было указано выше, однозначный клинический эффект применения “мост-терапии” у пациентов с высоким риском ТЭО до настоящего времени не подтвержден данными клинических исследований $[6,15,16]$. Напротив, описано увеличение частоты геморрагических событий на фоне "мосттерапии” у этой категории больных. Имеются отдельные сообщения о том, что у больных с протезами клапанов, имеющих высокий риск ТЭО: механические протезы митральных клапанов или поворотно-дисковые клапаны, может существовать относительно благоприятное соотношение частоты развития кровотечения и тромбозов на фоне назначения "мост-терапии”, однако эти результаты получены на основе только ретроспективного анализа наблюдений [21].

При необходимости могут использоваться и более консервативные стратегии проведения "мост-терапии" с применением низкодозированного гепарина, отсроченным началом его введения после операции, ранним переходом от гепарина к другим препаратам по мере приближения уровня MHO к показателю 2,0 [22, 23]. Следует также рассматривать возможность применения нефармакологических методов для снижения риска тромбоза, с целью чего используется ранняя активизация больных, а также ношение компрессионного трикотажа.

Перипроцедурная терапия с применением прямых пероральных антикоагулянтов (ППОАК)

В последнее время в периоперационном периоде все чаще применяются ППОАК. Временная отмена этих препаратов часто использовалась в рамках исследований RE-LY (Randomized Evaluation of Long-term Anticoagulation) - дабигатран vs варфарин, ROCKET-AF (Rivaroxaban Once 
daily oral direct Factor Xa inhibition Compared with vitamin $\mathbf{K}$ antagonism for prevention of stroke and Embolism Trial in Atrial Fibrillation) - ривароксабан vs варфарин, и ARISTOTLE (Apixaban for Reduction in Stroke and Other Thromboembolic Events in Atrial Fibrillation) - апиксабан vs варфарин, - в 25\%, 33\% и $34 \%$ случаях, соответственно [24]. Сроки временной отмены препаратов в зависимости от уровня риска кровотечения и клиренса креатинина отражены в таблице 5 [25].

У пациентов, включенных в эти крупные исследования, в т.ч. у тех, кому выполнялись крупные или экстренные вмешательства, не было выявлено повышенной частоты развития ТЭО или кровотечений в периоперационном периоде как при проведении "мост-терапии", так и в ее отсутствие. Учитывая сходство фармакокинетических характеристик этих препаратов и НМГ, по-видимому, применение ППОАК обладает лучшим безопасным потенциалом для ведения больных в периоперационном периоде, в сравнении с использованием варфарина [26].

\section{Заключение}

Одним из современных подходов к ведению пациентов, получающих АКТ в периоперационный период, является "мост-терапия" - вариант лечения, заключающийся в отмене ОАК с назначением НМГ или НФГ.

Возможность проведения “мост-терапии” целесообразно рассматривать у больных с высоким риском развития ТЭО без избыточного риска геморрагии, напротив, у пациентов с низким риском ТЭО

\section{Литература}

1. Liu S, Li X, Shi Q, et al. Outcomes associated with warfarin time in therapeutic range among US veterans with nonvalvular atrial fibrillation. Curr Med Res Opin. 2017; 25: 1-25. DOI: 10.1080/03007995.2017.1384370

2. Kolbin AS, Tatarskiy BA, Biserova IN, et al. Socio-economic burden of atria fibrillation in the Russian Federation. Clinical pharmacology and therapy 2010; 19 (4): 19-22. (In Russ.) Колбин А. С., Татарский Б. А., Бисерова И.Н. и др. Социальноэкономическое бремя мерцательной аритмии в Российской Федерации Клиническая фармакология и терапия. 2010; 19 (4): 19-22.

3. Serdechnaya EV, Kazakevich EV, Tatarskiy BA. Features of prevalence and course of atrial fibrillation in the Northwest of the Russian Federation. Clinical medicine 2009; 1: 17-20. (In Russ.) Сердечная Е. В., Казакевич Е. В., Татарский Б. А. Особенности распространенности и течения фибрилляции предсердий на Северо-западе Российской Федерации. Клиническая медицина 2009; 1: 17-20.

4. Nishtala A, Piers RJ, Himali JJ, et al. Atrial Fibrillation and Cognitive Decline in the Framingham Heart Study. Heart Rhythm. 2017; Sep 22. pii: S1547-5271(17)31133-5. DOI: 10.1016/j.hrthm.2017.09.036.

5. Guenancia C, Garnier F, Mouhat B, et al. Screening and clinical implications of silent atrial fibrillation. Rev Med Interne 2017; Sep 21. pii: S0248-8663(17)30632-X. DOI: 10.1016/j.revmed.2017.08.006.

6. Siegal D, Yudin J, Kaatz S, et al. Periprocedural heparin bridging in patients receiving vitamin $\mathrm{K}$ antagonists: systematic reviewand meta-analysis of bleeding and thromboembolic rates. Circulation 2012; 126 (13): 1630-9.

7. Gorenek B, Lip GY. Recent scientific documents from the European Heart Rhythm Association (EHRA). Europace 2015; 17 (8): 1161-3. DOI: 10.1093/europace/euv193.

8. Bansal VK, Herzog CA, Sarnak MJ, et al. Oral Anticoagulants to Prevent Stroke in Nonvalvular Atrial Fibrillation in Patients With CKD Stage 5D: An NKF-KDOQ Controversies Report. Am J Kidney Dis 2017; 70 (6): 859-68. DOI: 10.1053/j. ajkd.2017.08.003.

9. Denas G, Gennaro N, Ferroni E, et al. Effectiveness and safety of oral anticoagulation with non-vitamin $\mathrm{K}$ antagonists compared to well-managed vitamin $\mathrm{K}$ antagonists in “мост-терапия" не назначается. При рисках ТЭО и кровотечений средней степени следует проводить индивидуальную оценку возможности назначения “мост-терапии" в зависимости от специфических рисков этих осложнений, характерных как для пациента, так и предстоящей хирургической процедуры.

Схемы АКТ, включая отмену препаратов перед вмешательствами, следует применять строго в соответствии со стандартным протоколом, отклонения от которого в реальной клинической практике могут нивелировать преимущества “мост-терапии”, вследствие чего частота осложнений будет сходной с таковой без ее использования. Это подтвердили и предварительные данные настоящего исследования. Нередко в клинической практике отмечается неправильное назначение/отмена периоперационной АКТ: очень ранняя отмена ОАК до планируемой операции с назначением "мост-терапии", отмена ABK накануне оперативного лечения без назначения "мост-терапии", отсутствие мониторинга МНО до/после операции, возобновление приема ОАК только в день выписки с соответствующей отменой НМГ накануне.

Необходимы дальнейшие исследования клинической эффективности и безопасности различных схем периоперационной АКТ у пациентов, нуждающихся в плановом хирургическом лечении.

Конфликт интересов: все авторы заявляют об отсутствии потенциального конфликта интересов, требующего раскрытия в данной статье.

naive patients with non-valvular atrial fibrillation: Propensity score matched cohort study. Int J Cardiol 2017; 249: 198-203. DOI: 10.1016/j.ijcard.2017.09.029.

10. Vinereanu D, Al-Khalidi HR, Rao MP, et al. Regional differences in presentation and antithrombotic treatment of patients with atrial fibrillation: Baseline characteristics from a clustered randomized trial to IMProve treatment with AntiCoagulanTs in patients with atrial fibrillation (IMPACT-AF). Am Heart J 2017; 192: 38-47. DOI: 10.1016/j.ahj.2017.07.004

11. Lip GY, Laroche C, Popescu MI, et al. Improved outcomes with European Society of Cardiology guideline-adherent antithrombotic treatment in high-risk patients with atrial fibrillation: a report from the EORP-AF General Pilot Registry. Europace 2015; 17 (12): 1777-86. DOI: 10.1093/europace/euv269.

12. Rechenmacher SJ, Fang JC. Bridging Anticoagulation: Primum Non Nocere. JACC 2015; 66 (12): 1392-403. DOI: 10.1016/j.jacc.2015.08.002

13. Petit-Moneger A, Thiessard F, Noize P, et al. Definition of indicators of the appropriateness of oral anticoagulant prescriptions in hospitalized adults: Literature review and consensus (PACHA study). Arch Cardiovasc Dis 2017 Sep 21. pii: S18752136, (17)30154-7. DOI: 10.1016/j.acvd.2017.05.005.

14. Caprini JA, Traverso $\mathrm{Cl}$, Arcelus Jl. Perspectives on thromboelastography. Semin Thromb Hemost 1995; 21: 91-3.

15. WysokinskiWE, McBane RD. Periprocedural bridging management of anticoagulation. Circulation 2012; 126: 486-90. DOI: 10.1161/CIRCULATIONAHA.112.092833.

16. Clark NP, Witt DM, Davies LE, et al. Bleeding, recurrent venous thromboembolism, and mortality risks during warfarin interruption for invasive procedures. JAMA Intern Med 2015; 175: 1163-8. DOI: 10.1001/jamainternmed.2015.1843.

17. Brotman DJ, Streiff MB. Overuse of bridging anticoagulation for patients with venous thromboembolism: first, do no harm. JAMA Intern Med 2015; 175: 1169-70. DOI: 10.1001/jamainternmed.2015.1858.

18. Steinberg BA, Peterson ED, Kim S, et al. Use and outcomes associated with bridging during anticoagulation interruptions in patients with atrial fibrillation: findings from the Outcomes Registry for Better Informed Treatment of 
Atrial Fibrillation (ORBIT-AF). Circulation 2015; 131: 488-94. DOI: 10.1161/ CIRCULATIONAHA.114.011777.

19. Douketis JD, Spyropoulos AC, Kaatz S, et al. Perioperative bridging anticoagulation in patients with atrial fibrillation. N Engl J Med 2015; 373: 823-33. DOI: 10.1056/ NEJMoa1501035.

20. Douketis JD, Spyropoulos AC, Spencer FA, et al. Perioperative management of antithrombotic therapy: antithrombotic therapy and prevention of thrombosis, 9th ed: American College of Chest Physicians evidence-based clinical practice guidelines. Chest 2012; 141: 326-50. DOI: 10.1378/chest.11-2298.

21. Tafur AJ, McBane R, Wysokinski WE, et al. Predictors of major bleeding in periprocedural anticoagulation management. J Thromb Haemost 2012; 10: 2617. DOI: 10.1111/j.1538-7836.2011.04572.x.

22. Cavalcanti R, Rosenbaum B, Benzel E, Varma N. "Safe period" of anticoagulation withdrawal in patients with mechanical heart valve(s). JACC 2015; 65: A2035.

23. Jaffer AK, Brotman DJ, Bash LD, et al. Variations in perioperative warfarin management: outcomes and practice patterns at nine hospitals. Am J Med 2010; 123: 141-50. DOI: 10.1016/j.amjmed.2009.09.017.
24. Granger $\mathrm{CB}$, Lopes RD, Hanna M, et al. Clinical events after transitioning from apixaban versus warfarin to warfarin at the end of the Apixaban for Reduction in Stroke and Other Thromboembolic Events in Atrial Fibrillation (ARISTOTHLE) trial. Am Heart J 2015; 169: 25-30. DOI: 10.1016/j.ahj.2014.09.006.

25. Heidbuchel H, Verhamme P, Alings M, et al. Updated European Heart Rhythm Association Practical Guide on the use of non-vitamin $\mathrm{K}$ antagonist anticoagulants in patients with non-valvular atrial fibrillation. Eur Heart J 2017; 38 (27): 2137-49. DOI: 10.1093/eurheartj/ehw058.

26. Ezekowitz MD, Nagarakanti $\mathrm{R}$, Noack $\mathrm{H}$, et al. Comparison of Dabigatran and Warfarin in Patients With Atrial Fibrillation and Valvular Heart Disease: The RE-LY Trial (Randomized Evaluation of Long-Term Anticoagulant Therapy). Circulation 2016; 134 (8): 589-98. DOI: 10.1161/CIRCULATIONAHA.115.020950. 